\title{
Design and analysis of electric vehicle battery management system based on flexray bus
}

\author{
Jiangyi Lv ${ }^{1,2, a}$, Guanli Huang ${ }^{1}$, Hualei Zhang ${ }^{1}$, Jubiao Yao ${ }^{1}$ and Yong Chen ${ }^{2}$ \\ ${ }^{1}$ School of Automotive Engineering, Beijing Polytechnic , Beijing, China \\ ${ }^{2}$ School of Mechanical Engineering, Beijing Institute of Technology, Beijing, China
}

\begin{abstract}
Flexray bus is considered as a more promising bus in the future with the performance of real-time, scalable, and fault-tolerant. In this paper we design an electric vehicle battery management system based on flexray bus, including the hardware design, the SoC estimation theory, flexray protocol design and software development. The test bench experiment results show the system design is reasonable and feasible.
\end{abstract}

Keywords: flexray; BMS; li-ion battery; SoC.

\section{Introduction}

The state of power battery largely determines vehicle performance of electric vehicle. Lithium ion power battery with a nominal high voltage, high specific energy, long life and other advantages, is considered to be the future electric vehicles battery with very potential for development. It has been widely used in pure electric vehicles, hybrid vehicles and fuel cell vehicles [1]. But it still can not meet the requirements of current electric vehicle mileage. So power battery group and battery management system is particularly important. CAN bus is widely used in the automotive field, but for the electric car with long mileage, battery with more bus may cause insufficient bandwidth, and the real-time property of the battery management system wouldn't be good. The Flexray bus has high bandwidth (single channel up to $10 \mathrm{M}$ ), deterministic (time trigger) and fault tolerance (double channel redundancy) and other characteristics, will fully meet the demand of battery management system.

\section{Flexray bus protocol}

\subsection{The controller host interface $(\mathrm{CHI})$}

The controller host interface (CHI) deals with the management of data among host processor and each node of the FlexRay protocol engine. It also control the data transfer between them. CHI consists of two main modules: protocol data interface and data interface. Protocol data interface manages data exchange related to protocol operation, data interface manages all data related to information exchange [2]. The relationship between the architecture shown in Figure 1:

\footnotetext{
a Corresponding author : ljybuaa@163.com
} 


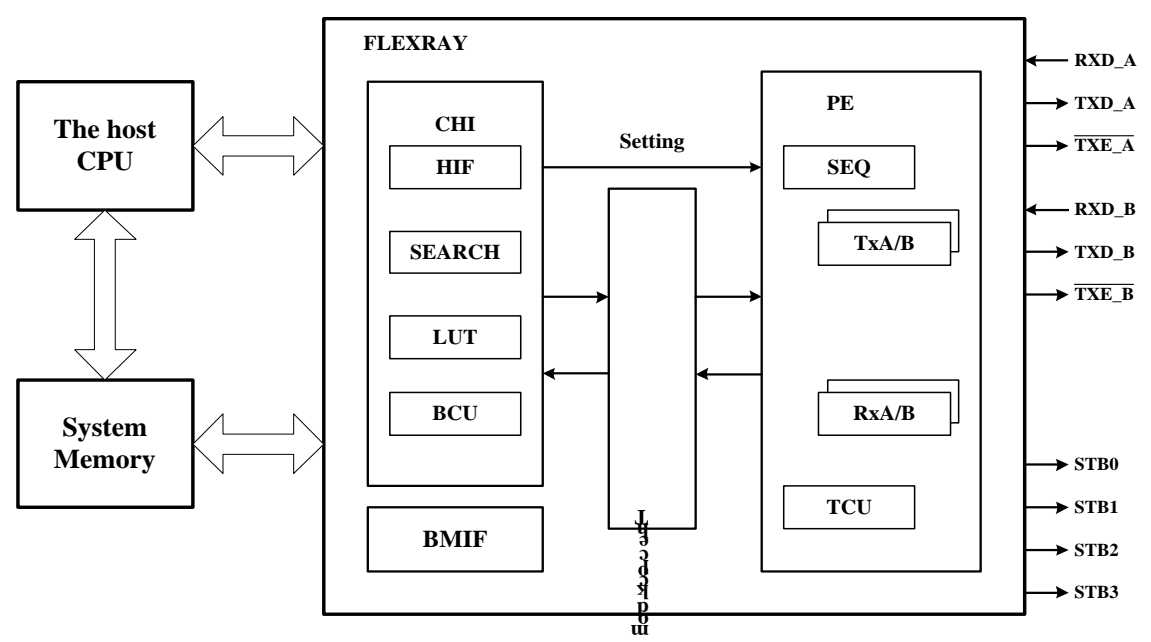

Figure 1. The concept structure of the controller's host interface

\subsection{Protocol operation control(POC)}

There are eight POC states of FlexRay protocol: the default configuration, configuration, ready, suspend, wake up, start, active and passive normal. The state-transition is shown in Figure 2 :

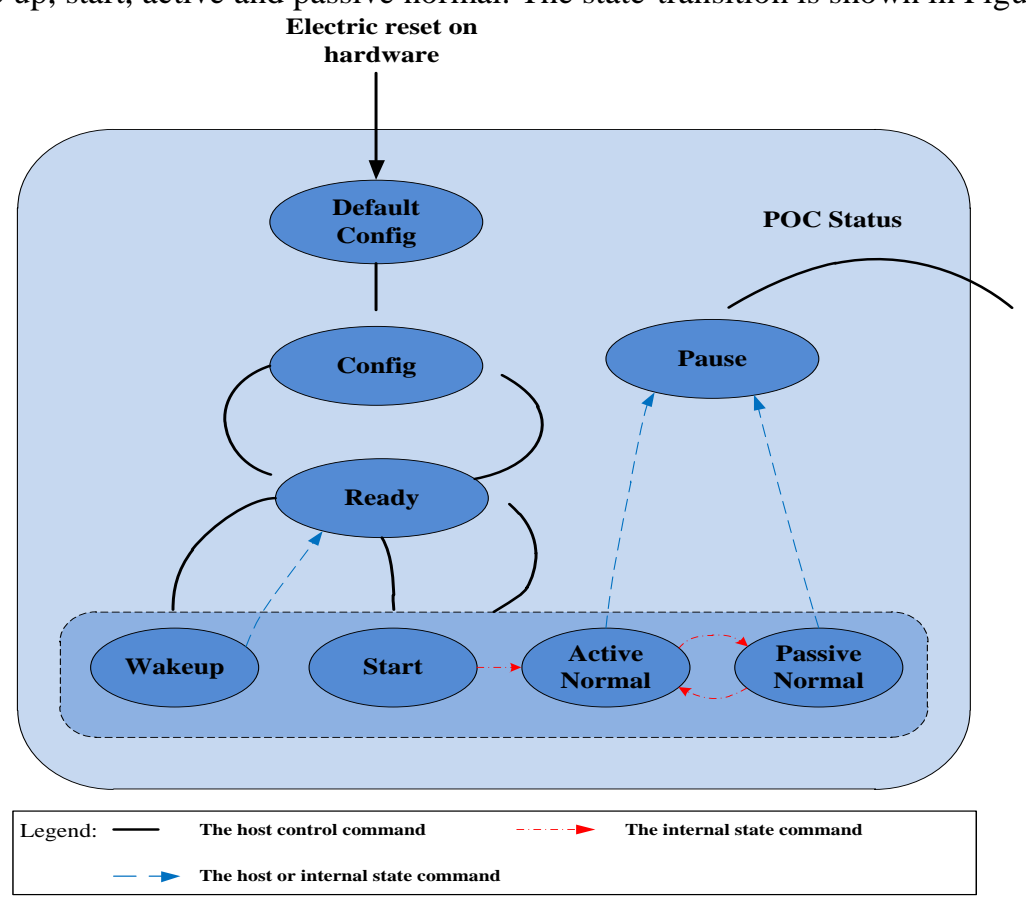

Figure 2. The POC agreement state-transition

\subsection{FlexRay data frame structure}

The structure of FlexRay data frame as shown in Figure 3, including: head section, load section and tail section. 


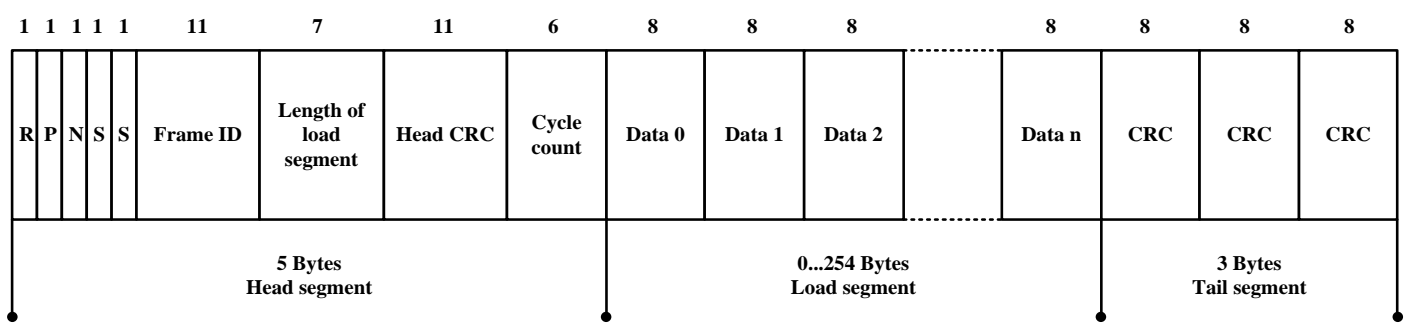

Figure 3. The data frame structure of FlexRay

\subsection{FlexRay media access control}

In FlexRay protocol, media access control is based on the communication cycle. In a communication cycle, FlexRay provides two kinds of media access scheme. One is static time division multiple access (TDMA), another is dynamic and more flexible time division multiple access (FTDMA) which based on the hour slot.

The communication cycle is the basic elements of FlexRay media access mechanism. Each communication cycle is composed of four parts: static and dynamic segment, symbol window and network idle time, the length of each part within the 10-16000us. It is defined based on the time level. The four time levels, from the bottom to the top are: minimum time layer, maximum time layer, arbitration grid layer and communication periodic layer. The structure shown in Figure 4:

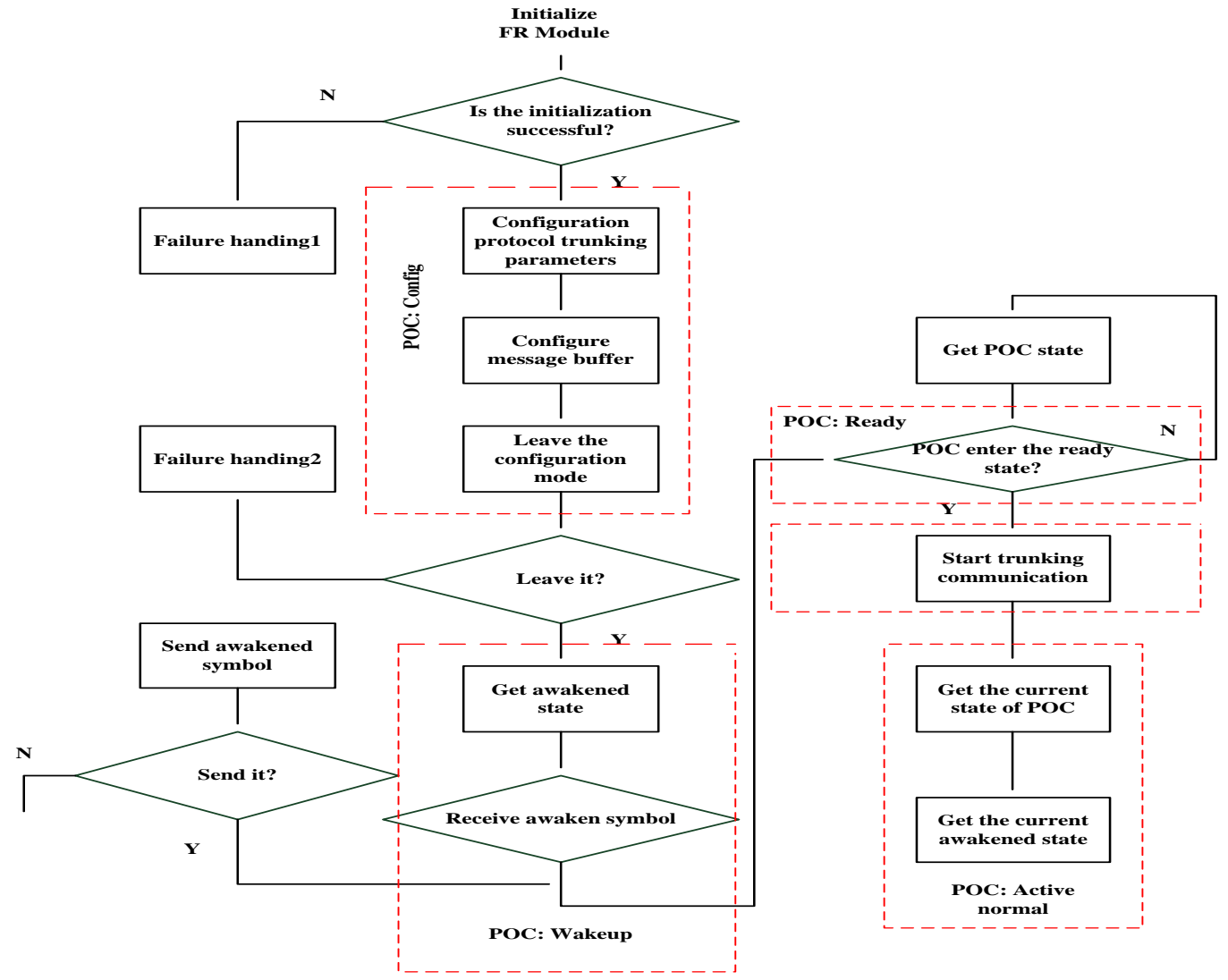

Figure 4. The communication cycle and time level of FlexRay 


\subsection{Time synchronization mechanism}

Time synchronization includes two main processes: modifying time synchronization (MTS) and computing synchronization time (CST). MTS control the time slot initial value, and modify it. CST mainly deals with communication cycle initialization, measurement and storage time deviation, calculating the frequency and phase value. The time deviation can be divided into offset and rate deviation. The offset is the absolute difference in a specific time between two clocks, rate deviation is the change of the offset with the passage of time. It reflects the change of the offset deviation at a particular time. The time synchronization algorithm modifies both offset and rate deviation.

\section{Design of battery management system}

\subsection{Design of battery management system hardware}

In the vehicle arrangement, because of the number of battery, battery box is usually installed, so this paper uses the distributed structure. This structure effectively avoids the traditional centralized acquisition signal when the measurement point is too much and detection circuit is complex. This structure also simplifies the hardware design of battery information acquisition channels, improves the anti-interference ability of signal acquisition.

\subsubsection{Acquisition module}

The acquisition module in a battery management system is divided into three different acquisition units: voltage acquisition, current acquisition and temperature acquisition.

There are two categories of series battery monomer voltage acquisition method. One is common mode measurement and another is differential mode measurement. This paper achieves monomer voltage acquisition by LTC6802. This chip supports a variety of battery module structure arrangement. Each chip measures at least 4 connected batteries with the monomer battery voltage. When the batteries are connected in series and the monomer battery number is less than 12, connecting the remaining $\mathrm{Cn}$ pin circuit will do. When measuring more than 12 single cell voltage, we can use the daisy chain structure to expand. The hardware structure principle is shown in Figure 5.

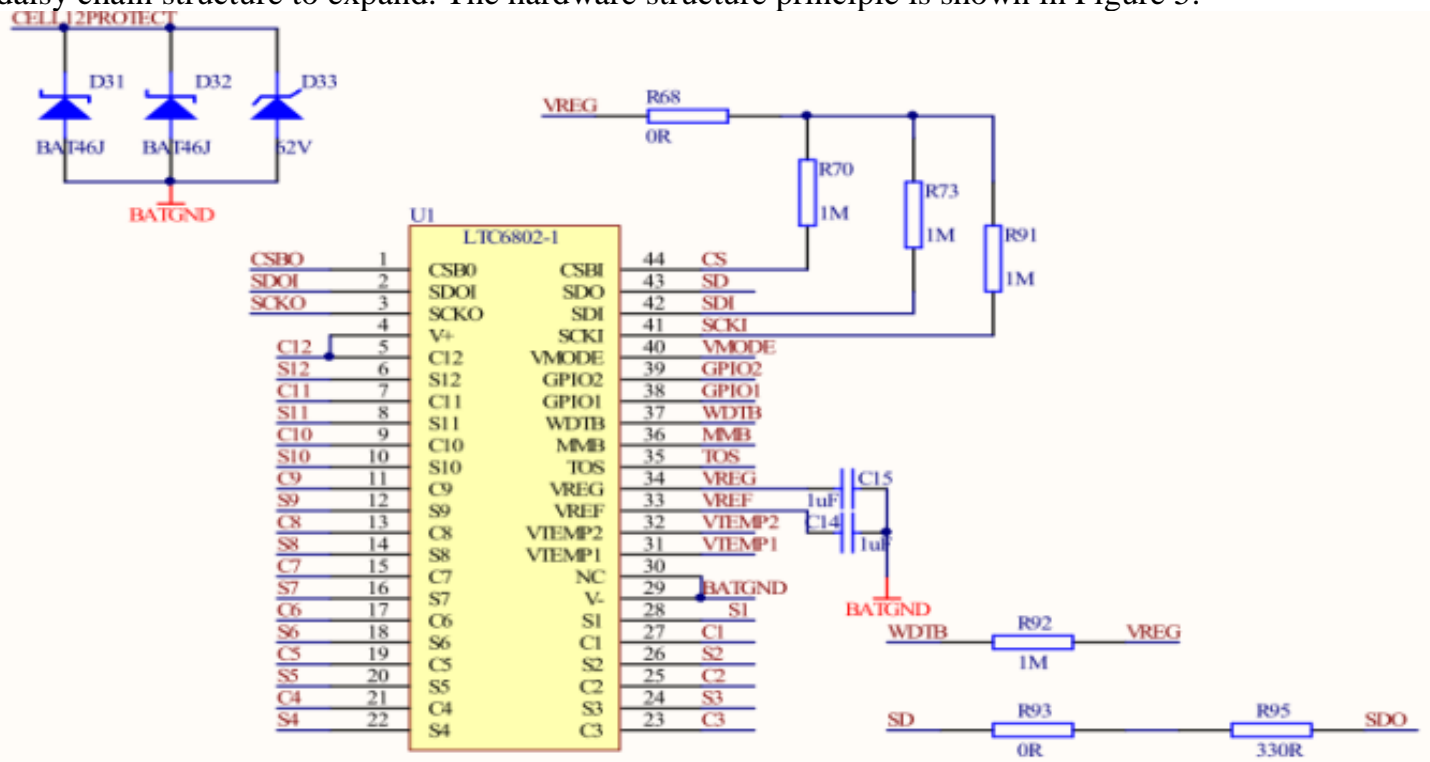

Figure 5. LTC6082 Theschematic diagram of voltage acquisition module 
DS18B20 is one of temperature sensor produced by Dallas company using one of the 1-wire bus communication mode. It has the advantages of simple structure, convenient operation, short conversion time, long transmission distance, high resolution and being not subject to outside interference. It is very suitable for building high precision, multi-point temperature measurement system. The hardware principle is shown in Figure 6.

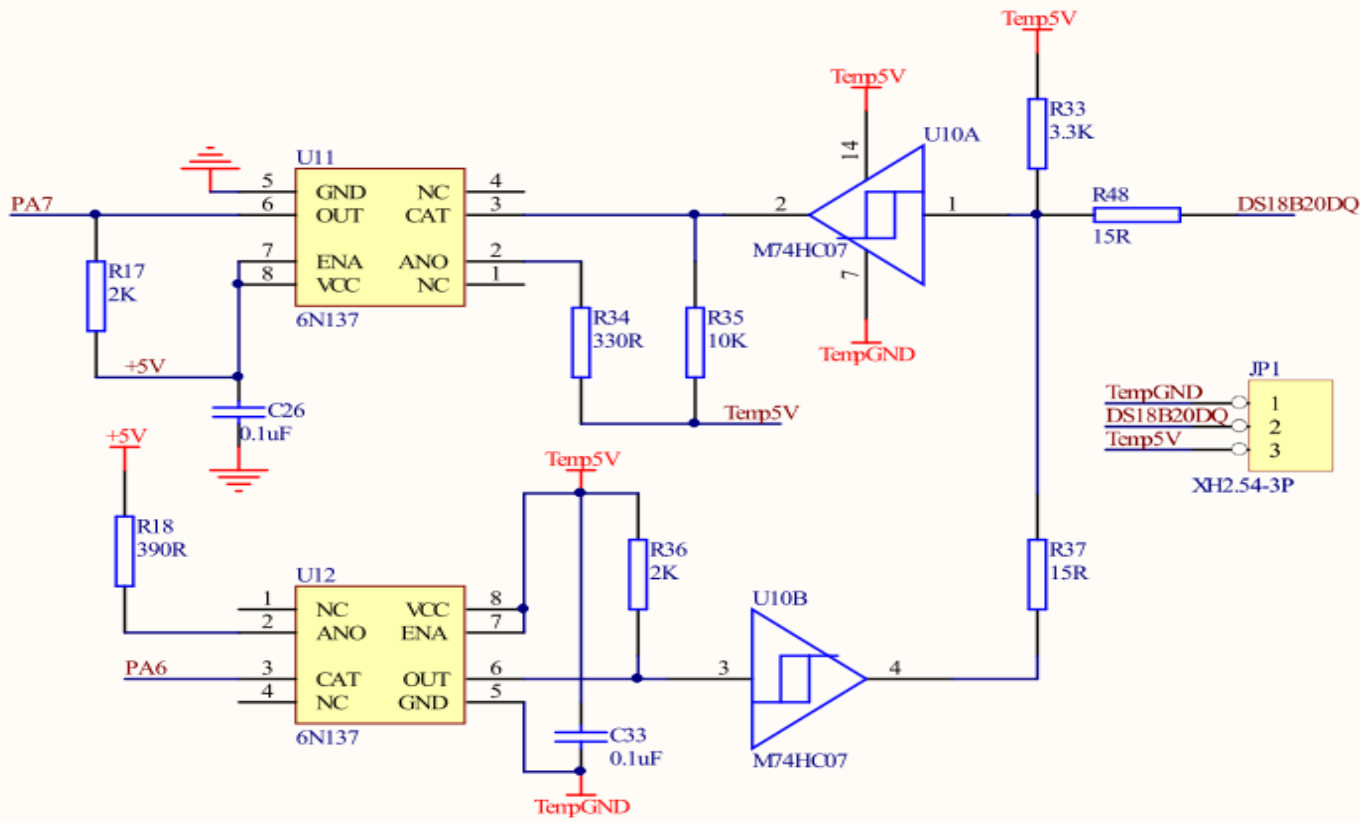

Figure 6. The schematic diagram of temperature acquisition module

\subsubsection{Flexray communication module}

Flexray implementation program has two forms. One is the use of single-chip MCU, independent communication controller CC and BD bus driver, another is the use of MCU integrated FlexRay function and bus driver BD. This paper adopts the Freescale MC9S12XF512 microcontroller integrated FlexRay communication controller CC, and bus driver TJA1080. The principle is shown in Figure 7.

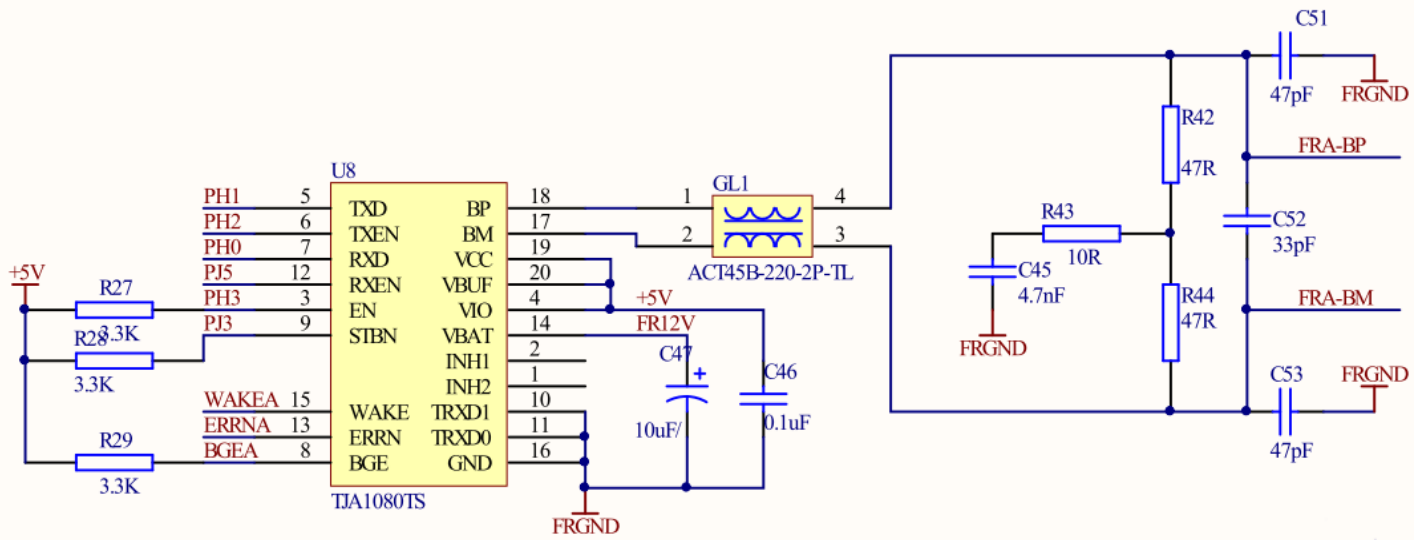

Figure 7. The schematic diagram of communication for FlexRay 


\subsection{Design of battery management system software}

\subsubsection{Flexray implementation of communication}

The FlexRay specification does not make a definition for the application layer. This paper developed a FlexRay network application layer protocol. The basic parameters of FlexRay network are configured which include: communication speed, communication cycle, static length, static slot length, dynamic segment length and hour slot length. According to the requirements of the safety and real-time of the battery management system, we use $10 \mathrm{Mbps}$ communication rate cluster to ensure rapid and detailed information transmission. We set appropriate parameters and write these parameters into vehicle spy FlexRay software, then corresponding simulation test is done with interpace FlexRay EVB board.

The results showed that the parameters configuration is reasonable. It realizes the normal operation of the network. Then we establish the corresponding communication task table according to needs. Table 1 is for the static segment frame definition, table 2 is for the definition of the dynamic segment frame:

Table 1. The ID definition of the static segment frame.

\begin{tabular}{cccccccc}
\hline $\begin{array}{c}\text { FR head segment } \\
\text { frame ID }\end{array}$ & ID10 to ID6 & ID5 & ID4 & ID3 & ID2 & ID1 & ID0 \\
\hline Specific meaning & 0 & retain & & Message number & & source address \\
\hline
\end{tabular}

Table 2. The ID definition of the dynamic segment frame.

\begin{tabular}{ccccccc}
\hline $\begin{array}{c}\text { FR head segment } \\
\text { frame ID }\end{array}$ & ID10 & ID9 & ID8 & ID7 & ID6 & ID5 to ID0 \\
\hline Specific meaning & priority & message type & message properties & 0 \\
\hline
\end{tabular}

\subsubsection{SoC estimation}

Accurate SoC estimation needs an accurate model of battery. The electrochemical model and equivalent circuit model has been widely used in the electric vehicle, so this paper selects the composite electrochemical model [3]. The model can be expressed as:

$$
y_{\mathrm{k}}=K_{0}+K_{1} S o C_{\mathrm{k}}+K_{2} / S o C_{\mathrm{k}}+K_{3} \ln \left(S o C_{\mathrm{k}}\right)+K_{4} \ln \left(1-S o C_{\mathrm{k}}\right)-R_{\mathrm{o}} I_{\mathrm{L}}
$$

Where yk denotes the end voltage of the model, R0 denotes internal resistance, K0, K1, K2, K3, K4, K5 are 6 constants associated with the model.

This paper use unscented Kalman filter algorithm for state estimation. Unscented Kalman filter uses linear Kalman filter framework, based on unscented transformation, through the deterministic sampling strategy to approximate nonlinear distribution.

This paper uses central sampling strategy, determine the weights and define as follows:

$$
\begin{array}{ll}
x_{0}=\bar{x}, & w_{0}^{m}=\lambda /(n+\lambda) \\
x_{i}=\bar{x}+\left(\sqrt{(n+\lambda) P_{x}}\right)_{i}, i=1,2 \ldots \ldots . n & w_{0}^{c}=\lambda /(n+\lambda)+\left(1-\alpha^{2}+\beta\right) \\
x_{i}=\bar{x}-\left(\sqrt{(n+\lambda) P_{x}}\right)_{i}, i=n+1, \ldots \ldots .2 n & w_{i}^{m}=w_{i}^{c}=1 / 2(n+\lambda), \quad i=1,2, \ldots, 2 n
\end{array}
$$

The state estimation process using UKF as follows:

(1) acquisition of Sigama sampling and weights;

(2) transfer of sampling points using the state equations;

(3) calculate the forecasting mean and covariance using the prediction of sampling points and weight;

(4) predict measurement sampling point using (2); 
(5) forecast measurement value and covariance;

(6) calculate the UKF gain and update state vector and variance.

\section{The test results and analysis}

In order to verify the real-time, accuracy and reliability of the battery management system, we have had the bench test, including: battery management system, the power load test, power battery t, ARBIN power battery test instrument, the host computer PC and a FlexRay EVB, etc. We take 35Ah/10 manganate lithium ion battery package as the test object. The accuracy of the battery management system and real-time data can be displayed by PC. The test platform is shown in Figure 8 . Monomer voltage data of the battery pack are shown in Table 3. The test results show that, the designed battery management system has high precision and meets the application requirements.

Table 3. Battery pack monomer voltage test results.

\begin{tabular}{lcc|lcc}
\hline number & sampling value $/ \mathrm{V}$ & measured value $/ \mathrm{V}$ & number & sampling value /V & measured value /V \\
\hline $\mathbf{1}$ & 3.324 & 3.323 & 6 & 3.327 & 3.325 \\
\hline $\mathbf{3}$ & 3.326 & 3.324 & 7 & 3.322 & 3.323 \\
\hline $\mathbf{3}$ & 3.324 & 3.322 & 8 & 3.324 & 3.321 \\
\hline $\mathbf{5}$ & 3.328 & 3.330 & 9 & 3.322 & 3.323 \\
\hline
\end{tabular}

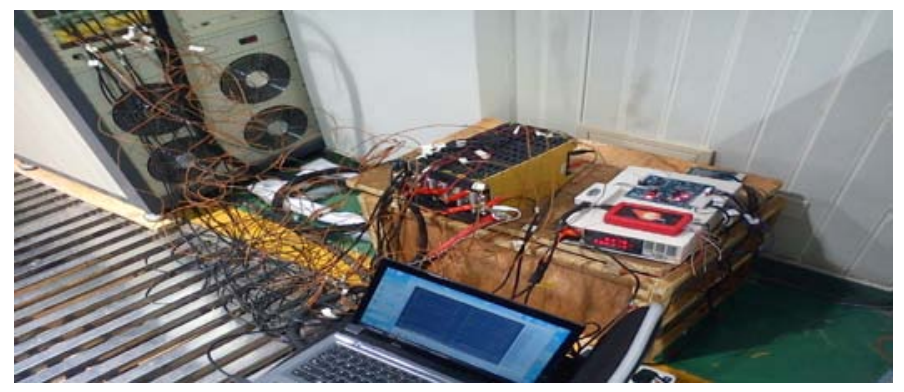

Figure 8. Battery management system test platform

\section{Conclusions}

The Flexray bus has characteristics of high bandwidth (single channel up to 10M), deterministic (time trigger) and fault tolerance (double channel redundancy), etc. It is very suitable for the application requirements of battery management system. This paper based on the analysis of FlexRay bus protocol and electric vehicle battery management system, completed the FlexRay bus and battery management system hardware design and software design. We carry out the experiment verification, the contrast analysis of the battery pack monomer voltage test data shows that the designed battery management system has high precision and highly meets the application requirements.

\section{Acknowledgments}

This work was supported by Program for Research Team in Beijing Polytechnic: YZK2013025.

\section{References}

1. Xiong Rui, He Hongwen, Ding Yin. Parameter identification method of HEV lithium ion battery dynamic model [J]. Power electronics technology, 45 (4): 100-102(2011) 
2. Fu Xingfeng, Research and verification of the electric vehicle battery safety management, Automotive technology,9:737-740(2013)

3. Xu Yongli, Application of Flexray bus in electric vehicle[D]. Beijing: Beijing Institute of Technology, (2012)

4. Xu Yong-li, HE Hong-wen. Design on data acquisition system for BMS used in electric vehicles [J]. Journal of Beijing Institute of Technology, 20 (Suppl.2): 220-224(2011)

5. Pei Feng, Research of 18650 battery power system safety design based on air cooling mode , Automotive technology, 8:253-256(2015)

6. Huang Guanli, Design of comprehensive traffic information processing based on multi point GPS, Computer measurement and control, 19 (12), 3041-3043(2011)

7. Bi Jun, Estimate of the electric vehicle battery SOC by artificial immune particle filter algorithm, Transportation systems engineering and information, 5:103-108(2015)

8. Hu Chunhua, Estimate of the electric vehicle lithium battery SOC based on PID neural network, Automotive technology, 10:36-38(2012)

9. Huang Guanli, Lv Jiangyi, Dynamic path optimization of traffic flow control and simulation, measurement and control technology, 1 (33), 148-153(2014)

10. Yang Jianjun, Research of the automobile disassembling course based on engineering applied talents training, Experimental technology and management, (02) 192-194(2014)

11. Fan bin, Finite element and fatigue life analysis of the hydrogen storage bottle of fuel cell in city bus, Automotive technology, 1:4-8(2013)

12. Bi Jun, A modeling and the correlation analysis between electric vehicle mileage and the battery SOC, Transportation systems engineering and information, 1:103-108(2015)

13. Yin Haitao, Research and design of intelligent automatic monitoring system for the vanadium redox flow battery , Experimental technology and management, (02) 45-47(2006) 